\title{
Mantenedor de Espacio Estético - Funcional en Odontopediatría: Reporte de caso
}

\author{
Maintainer of Aesthetic Space - Functional in Pediatric Dentistry. Case \\ Report
}

\author{
Sandra Echevarría-Mendieta ${ }^{1}$, Mariela Romero-Velarde ${ }^{2}$, Rita Villena -Sarmiento ${ }^{3}$
}

\section{RESUMEN}

La pérdida precoz de dientes deciduos es un problema común en odontopediatría y puede estar asociada a traumas en la región anterior a caries de infancia temprana (CIT), erupción ectópica entre otras y puede acarrear daño oclusal y otras secuelas estético-funcionales indeseables. La confección de un aparato mantenedor de espacio estético - funcional, es una de las opciones de tratamiento más utilizadas en este tipo de casos. Dentro de los principales aparatos mantenedores de espacio se encuentra la prótesis parcial anterior de sistema tubo-barra tipo Denari, entre las indicaciones de este, está la recuperación funcional, fonética y estética a fin de prevenir trastornos emocionales en los niños, mejorar el aspecto social y preservar las dimensiones correctas de la arcada, evitando desarmonías oclusales. Este relato de caso clínico muestra la importancia del mantenedor estético - funcional, su correcta confección y aplicación clínica en la primera infancia, orientando a los profesionales que atienden pacientes pediátricos.

Palabras Clave: Caries; Infancia temprana; Mantenedor de espacio; Prótesis fija. (Fuente: DeCS BIREME)

\section{ABSTRACT}

Early loss of deciduous teeth is a common problem in pediatric dentisty, can be associated with traumas of the anterior region of early childhood caries (ECC), ectopic eruption among others, and can cause occlusal damage and other undesirable aesthetic-functional squeal. The preparation of a functional - aesthetic space maintainer device is one of the most used treatment options in this type of condition; Among the indications of this, is the functional, phonetic and aesthetic recovery in order to prevent emotional disorders in children, improve the social aspect and preserve the correct dimensions of the arch, avoiding occlusal disharmonies. Within the main space mantainer devices we the partial anterior prosthesis of the Denari tube-bar system. This clinical case report shows the importance of the esthetic - functional maintainer, the correct preparation and clinical application in early childhood, orienting the professionals who treat pediatric patients.

Key words: Caries; Early childhood caries; Space maintainer; Fixed prosthesis. (Source: MeSH NLM)

${ }^{1}$ Universidad de San Martin de Porres. Lima, Perú.

a Residente de Posgrado de Odontopediatría

b Docente de posgrado en la Especialidad de Odontopediatría

\section{Correspondencia:}

Sandra Echevarría-Mendieta

Correo electrónico: cdechevarria@hotmail.com'

Citar como: Echevarría-Mendieta S, Romero-Velarde M, Villena Sarmiento R. Mantenedor de Espacio Estético - Funcional en Odontopediatría: Reporte de caso. KIRU. 2019; 16(2): 81 - 91. https://doi.org/10.24265/kiru.2019.v16n2.05 


\section{INTRODUCCIÓN}

Cuando un niño pierde dientes precozmente en el sector anterior en cerca del $70 \%$ de los casos de niños menores de 3 años es por trauma dental, el $30 \%$ restante se debe a la presencia de caries de infancia temprana (CIT), junto a los hábitos de alimentación cariogénica e higiene deficiente, dientes ectópicos entre otros factores asociados. ${ }^{1}$

La integridad de la dentición tiene influencia en la conservación de la oclusión, la estética, la fonética, la masticación y bienestar psicológico del niño. A lo largo de la primera infancia los niños se desenvuelven emocional, cognitiva, y físicamente, a través del aprendizaje y sociabilización, cuando el niño presenta una pérdida precoz de los dientes anteriores, se torna introspectivo, causando daño en el desenvolvimiento social(2). Así mismo esta pérdida prematura puede traer como consecuencias alteraciones morfológicas en la arcada dentaria, problemas de fonación,(3) esto conlleva a una preexistencia de hábitos, anomalías en la musculatura bucal, masticación, deglución y estética $^{(4)}$. Ahora en la mayoría de los casos de pérdida precoz de los dientes anteriores donde la oclusión aún no se ha establecido (antes de la erupción de los caninos deciduos), no muestra una pérdida de espacio significativo ${ }^{(5)}$.

Los padres acuden a los servicios odontológicos buscando tratamiento, cuando los niños presentan un cuadro inflamatorio agudo o cuando todas las coronas están destruidas, las necesidades estéticas van siendo cada vez más relatadas por los propios niños ${ }^{(6)}$.

La función masticatoria se ve alterada dependiendo de la cantidad de piezas perdidas, ya que los niños se adaptan a la ausencia de dientes y modifican su dieta para la deglución de alimentos pastosos, desarrollando una deglución atípica, el desenvolvimiento del habla se ve comprometida por la dificultad de pronunciar los sonidos de ciertas vocales, ya que la lengua toca las facetas palatinas de los incisivos superiores en una inapropiada compensación del habla, durante la emisión de los fonemas: /T/, /D/, /N/ y /L/,ocurre dificultades para la articulación de palabras y sonidos sibilantes como los fonemas :/S/ o /Z/. Una óptima opción para una sustitución de dientes perdidos es la confección de una prótesis funcional (7) pues generalmente son niños menores de 5 años, que tienen la capacidad de usar un aparato mantenedor de espacio removible o planeamiento de una prótesis fija para infantes, donde se debe considerar el crecimiento y desenvolvimiento de los niños ${ }^{(6,8,9)}$.

Existen diferentes tipos de tratamiento para reponer la pérdida prematura de las piezas deciduas si hablamos del sector anterior, entre ellas tenemos las prótesis fijas, semi-fijas y removibles, en tanto que en el sector posterior son los mantenedores de espacio tipo banda- ansa, arco de Nance, zapatilla lingual, etc. Es así que surge como opción de recurso estético - funcional en niños poco colaboradores, cuando son comparados con aparatos removibles, las prótesis fijas con cursor, conocida como prótesis tipo Denari (10) (Tabla 1), que no altera el crecimiento de los arcos dentales en los niños preconizada por Denari \& Correa en el año $1995^{(10)}$, posibilita su uso antes de la época de exfoliación de los elementos de soporte, se observa una abertura del sistema "tubo - barra" debido al crecimiento del maxilar permitiendo por tanto el crecimiento en lateralidad natural, diferente a la prótesis fija directa que no posee este sistema ${ }^{(10-13)}$.

Tabla 1. Características e indicaciones para recomendar un mantenedor fijo en odontopediatría.

\begin{tabular}{|c|c|c|c|c|}
\hline Accesorios & Póntico & Efectividad & Indicaciones & Limitaciones \\
\hline $\begin{array}{c}\text { Sistema } \\
\text { Tubo - } \\
\text { Barra }\end{array}$ & $\begin{array}{l}\text { Diente fabricado en } \\
\text { resina compuesta. } \\
\text { Diente de acrílico }\end{array}$ & $\begin{array}{l}\text { No requiere } \\
\text { cambios } \\
\text { periódicos. } \\
\text { Son } \\
\text { mínimamente } \\
\text { invasivos. }\end{array}$ & $\begin{array}{l}\text { Áreas edéntulas de } \\
\text { pequeña extensión. } \\
\text { Dientes de soporte no } \\
\text { cariado con o sin } \\
\text { pequeñas restauraciones. } \\
\begin{array}{l}\text { Dientes de soporte sin } \\
\text { movilidad. }\end{array}\end{array}$ & $\begin{array}{l}\text { Costo de laboratorio. } \\
\text { Cuidado con la higiene oral. } \\
\text { Remoción del dispositivo en } \\
\text { el tiempo adecuado. } \\
\text { Control radiográfico periódico. }\end{array}$ \\
\hline
\end{tabular}




\section{Objetivo}

Mostrar al cirujano dentista que trabaja con pacientes pediátricos la importancia de emplear el mantenedor estético - funcional. Esta prótesis presenta entre los incisivos centrales un cursor con un anclaje tipo "hembra - macho", que se va abriendo a medida que el maxilar va creciendo en su desenvolvimiento, es importante relatar que hay necesidad de desgastar los dientes para encajar la prótesis, este sistema ha sido desarrollado, como una opción para la pérdida precoz de dientes anteriores deciduos.

\section{DESCRIPCIÓN DEL CASO CLÍNICO}

Paciente de sexo masculino de 3 años de edad, que acude a la clínica especializada en odontopediatría, de la universidad "San Martin de Porras", en compañía de su madre para evaluación odontológica.

Durante la anamnesis la madre del paciente manifiesta su preocupación por las múltiples lesiones que presenta el niño, que afectan la estética y el posible compromiso emocional que puede acarrear, la madre refiere que su niño es un paciente temeroso con experiencias desagradables en procedimientos odontológicos anteriores.

Al examen clínico general el paciente presenta peso y talla inferiores al promedio de acuerdo a su edad, como antecedente medico el niño presento bronquitis de manera continua.

A la evaluación clínica extraoral se observa alteraciones en la fonoarticulación, deglución atípica, hábito de interposición lingual, perdida de dimensión vertical, a la evaluación intraoral amplia destrucción coronal en múltiples piezas.

El paciente fue sometido a exámenes complementarios como radiografías periapicales, radiografía panorámica e interconsultas a los servicios pertinentes, con el fin de confirmar el diagnóstico y realizar el plan de tratamiento.

El diagnóstico definitivo fue el siguiente:

Caries de Infancia Temprana, (CIT), con presencia de lesiones no cavitadas activas: Piezas 5.5, 5.3, 6.3, 6.4, 6.5, 7.5, 7.3, 7.2, 7.1, 8.1, 8.2, 8.3, 8.4, 8.5 (V). Lesiones cavitadas activas: 54 (OV), 5.3 (VP) ,6.3 (VP), 6.4(O), 6.5(O), 7.5(O), 8.5(O).

Pulpitis reversible: piezas 7.4, 8.4.

Pulpitis irreversible: piezas 5.2, 6.1, 6.2. Necrosis pulpar: Piezas 5.1.

Se hizo una evaluación de riesgo estomatológico del paciente, detectando un alto riesgo a nuevas lesiones de caries dental debido a un prolongado uso del biberón, dieta altamente cariogénica y una deficiente higiene dental, además se determinó una conducta definitivamente negativa según la escala de Frankl. (Figura 1, 2 y 3 ).
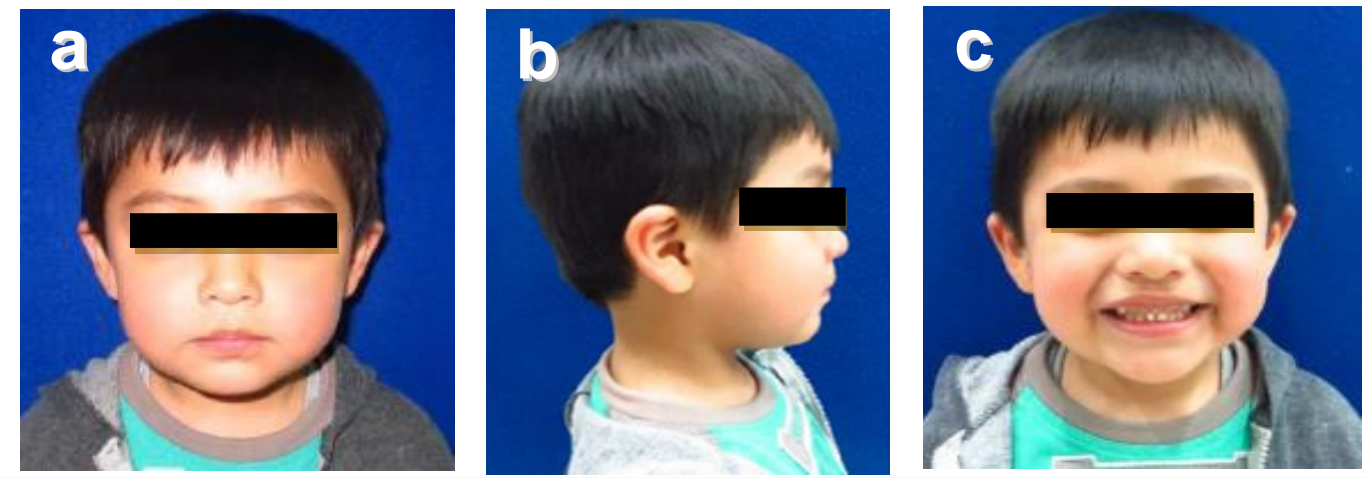

Figura 1. Fotos faciales pre -tratamiento: a) frontal b) perfil c) sonrisa
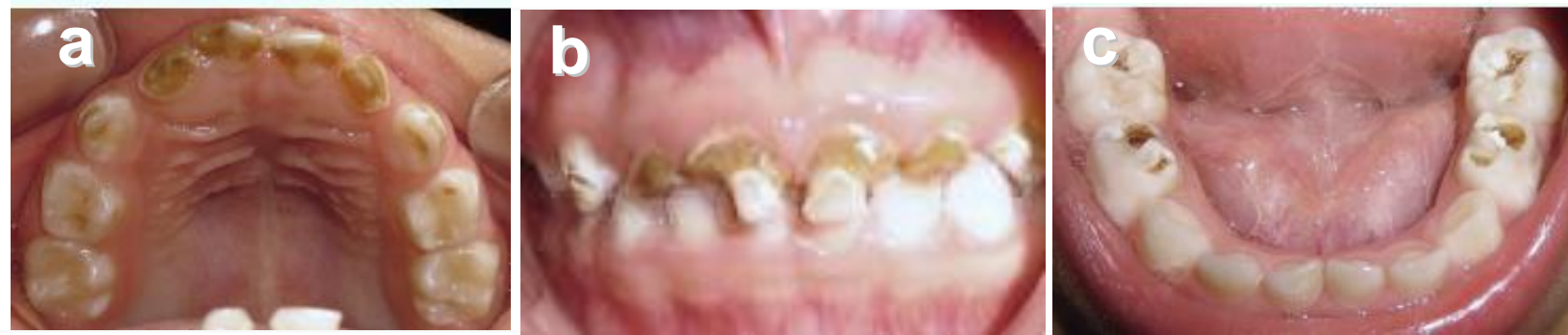

Figura 2. Fotos intraorales pre - tratamiento: a) vista palatina $\quad$ b) vista frontal c) vista lingual. 

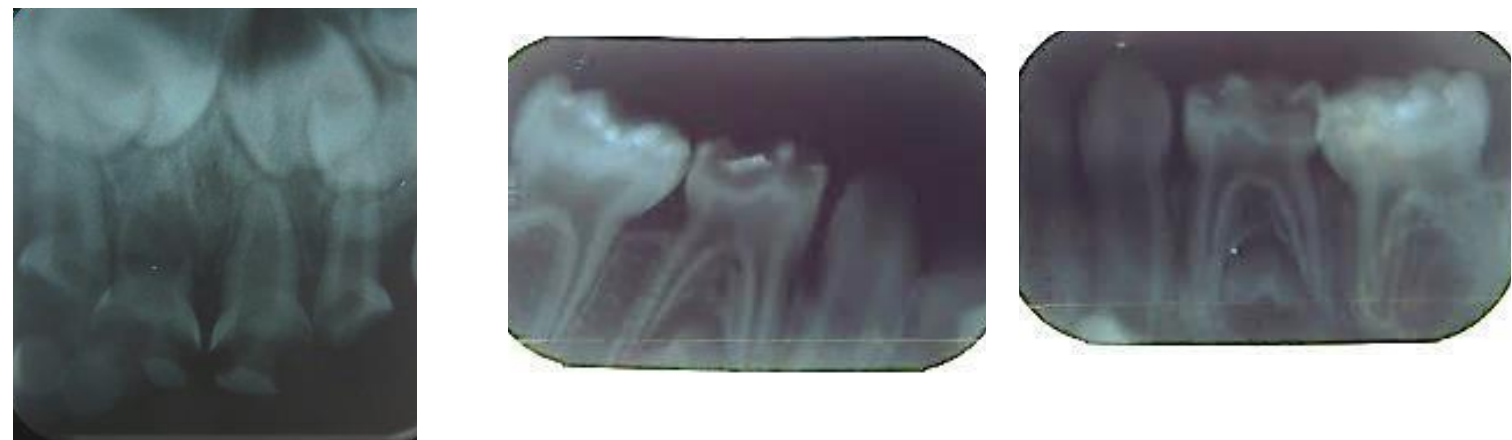

Figura 3. Radiografías de diagnóstico: a) Pzas.5.1, 6.1 b) Pzas. 8.4,8.5 c) Pzas.7.4, 7.5.

Por lo que se planificó tratamientos preventivos y restauradores que incluían: Profilaxis, Flúor terapia con Flúor barniz al 5\% (22600ppm de F). El paciente es ingresado a sala de operaciones (SOP) bajo anestesia general para realizar los siguientes tratamientos en el año 2014: Pulpectomías: Piezas 5.2, 6.1, 6.2 lonómero modificado de resina: $5.2,6.1,6.2$, debido a caries extensa y Exodoncia: Pieza 5.1

El paciente reingresó al servicio de odontopediatría al año siguiente 2015, para continuar con los tratamientos previamente planificados en SOP: Postes de fibra de vidrio en las piezas: $5.2,6.1,6.2$ bajo aislamiento relativo. lonómero modificado con Resina: piezas 5.4, 7.4,
7.5, 8.5, 6.3, 5.3. Restauraciones con Resina: piezas 5.2, 6.1, 6.2, Pulpotomías: piezas 7.4, 8.4, 8.5, Pulpectomías: pieza 7.5. Cuatro meses después el paciente presenta una reabsorción de la pieza 6.1, motivo por el cual se procede a la exodoncia. (Figura 4 y 5 )

Se realizaron impresiones para confección de modelos de estudio. (Figura $N^{\circ} 6$ ). Para la fase rehabilitadora se confeccionó coronas de ivocron para el sector posterior en las piezas que lo requerían, y el sector antero superior, fue rehabilitado mediante el uso de un dispositivo estético - funcional fijo tipo Denari (10).

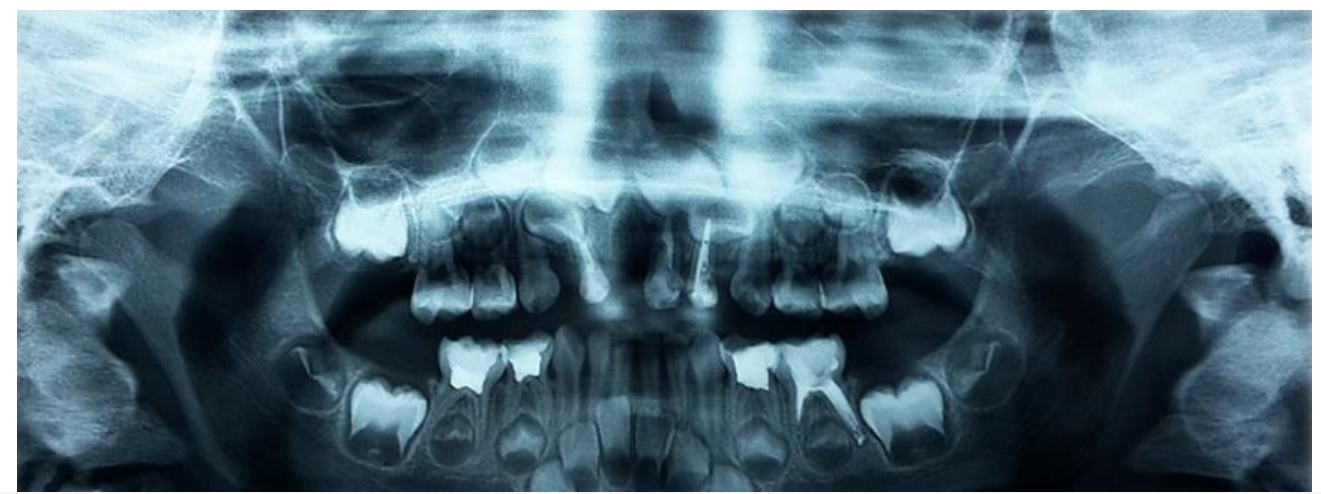

Figura 4. Radiografía panorámica pos -tratamiento año 2015, previo al dispositivo estético - funcional tipo Denari y mantenedor de espacio inferior.
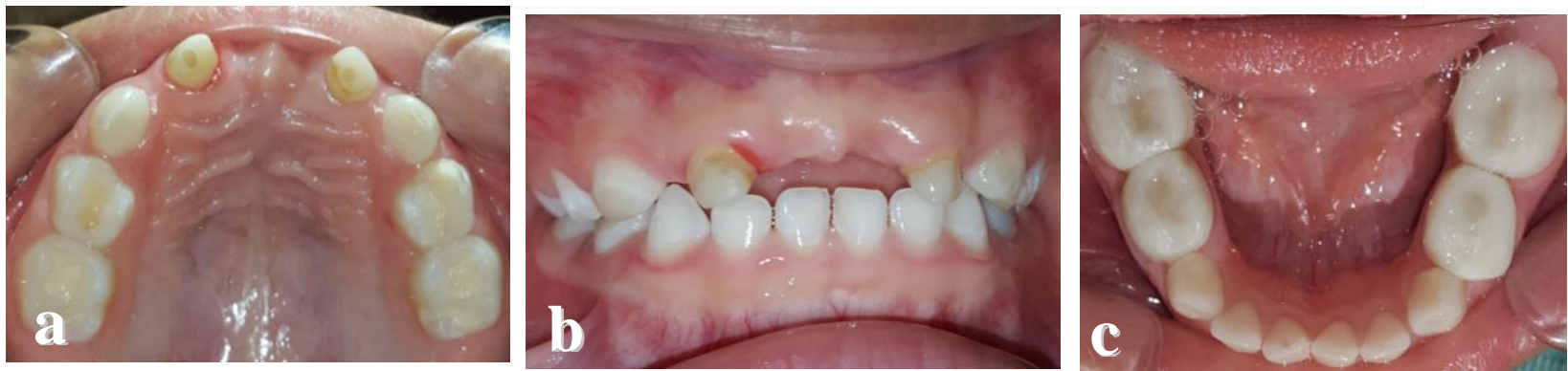

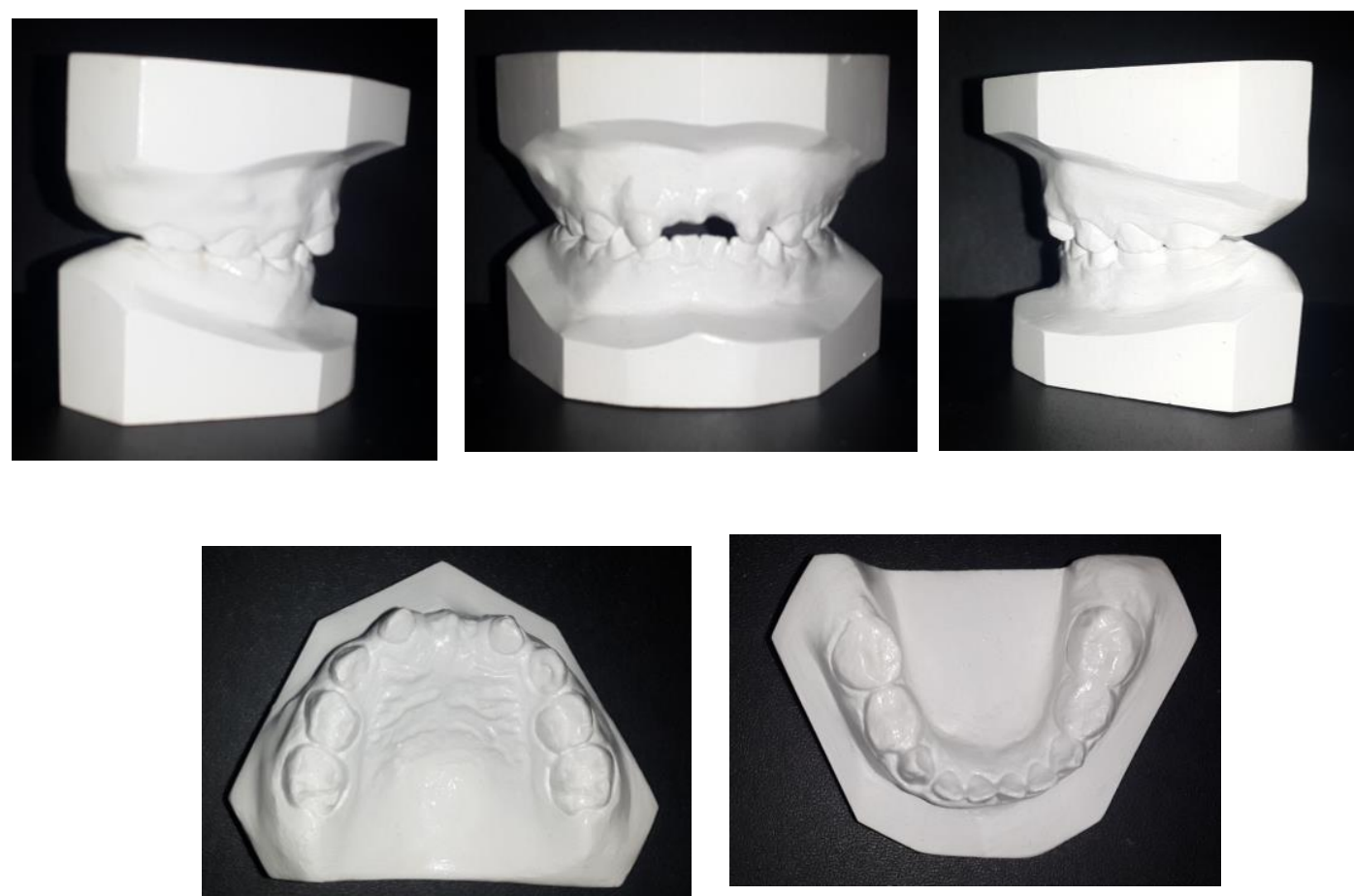

Figura 6. Modelos de estudio

Este consiste en una prótesis fija con pónticos de resina acrílica para reemplazar los dientes 5.1 y 6.1 un vástago de barra central y anclaje a los dientes 5.2 y 6.2. El vástago central el cual es rígido se conecta a un canal presente en uno de los pónticos, este tiene como función darle dinamismo a la prótesis, permitiendo el distanciamiento lento entre el retenedor y el póntico mediante el desplazamiento a través de la barra, esto permite acompañar el crecimiento transversal de la premaxila. Dado que se está reemplazando dos dientes, se decidió hacer una modificación del diseño original, preparando la estructura en su totalidad de Ivocron (resina acrílica), mejorando la estética de la prótesis, dicho dispositivo permitió levantar la mordida, devolver la dimensión vertical y restablecer la función estética del paciente. (Figura 7)

Antes de la confección del aparato protésico se realizó un tratamiento preventivo a fin de disminuir el riesgo de sufrir nuevas lesiones de caries y mejorar el control del biofilm, requisito esencial para cualquier portador de aparato protésico.
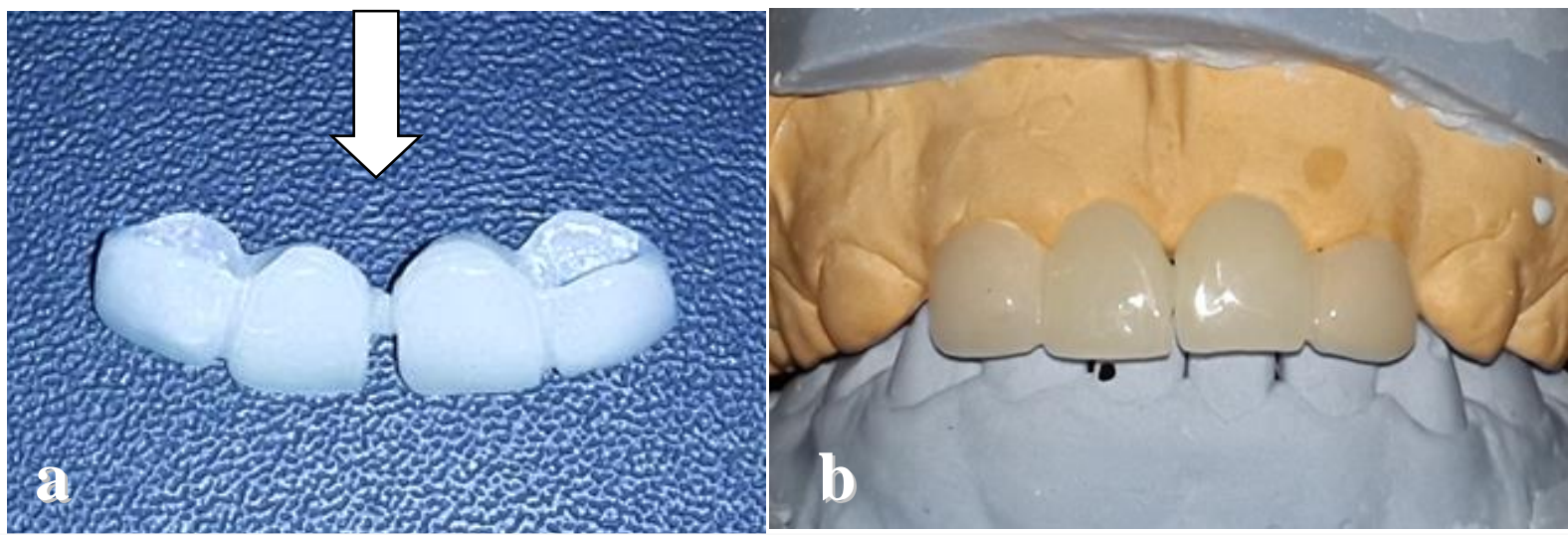

Figura 7. Prótesis fija estético - funcional tipo Denari: a) apreciamos el sistema tubo-barra.

b) modelo con la próstesis fija en posición -vista anterior 
Posteriormente se tomaron los modelos de las arcadas superior e inferior con silicona, el registro de mordida fue tomado con una lámina de cera, se optó por realizar una preparación en los dientes de apoyo previamente tratados. Durante el procedimiento el niño presentó un buen comportamiento, gracias al manejo de conducta realizado durante las diferentes fases de tratamiento.

Antes de cementar la prótesis, las dos partes del aparato fueron amarradas con hilo dental para evitar riesgo de aspiración y /o deglución de la prótesis. El aparato y los pilares fueron previamente higienizados con clorhexidina al $2 \%$, el vástago central fue insertado en el tubo presente en el póntico previa a la cementación de la prótesis e insertado por coincidencia del eje de inserción en los pilares. El sistema tubo - barra se encuentra previamente aliviado para permitir las posibles variaciones en el momento de la insertar la prótesis.

La (Figura 8) muestra la preparación de los pilares y la correcta inserción del dispositivo, cementada con cemento Allcem de polimerización dual FGM, donde se aprecia una vista frontal mostrando un buen ajuste sin daño en los tejidos blandos adyacentes.

La (Figura 9) muestra una vista frontal y palatina de la prótesis luego de 8 meses de seguimiento, donde se destaca un aumento de espacio interincisivo de aproximadamente $1.2 \mathrm{~mm}$, lo que demuestra un incremento en sentido transversal
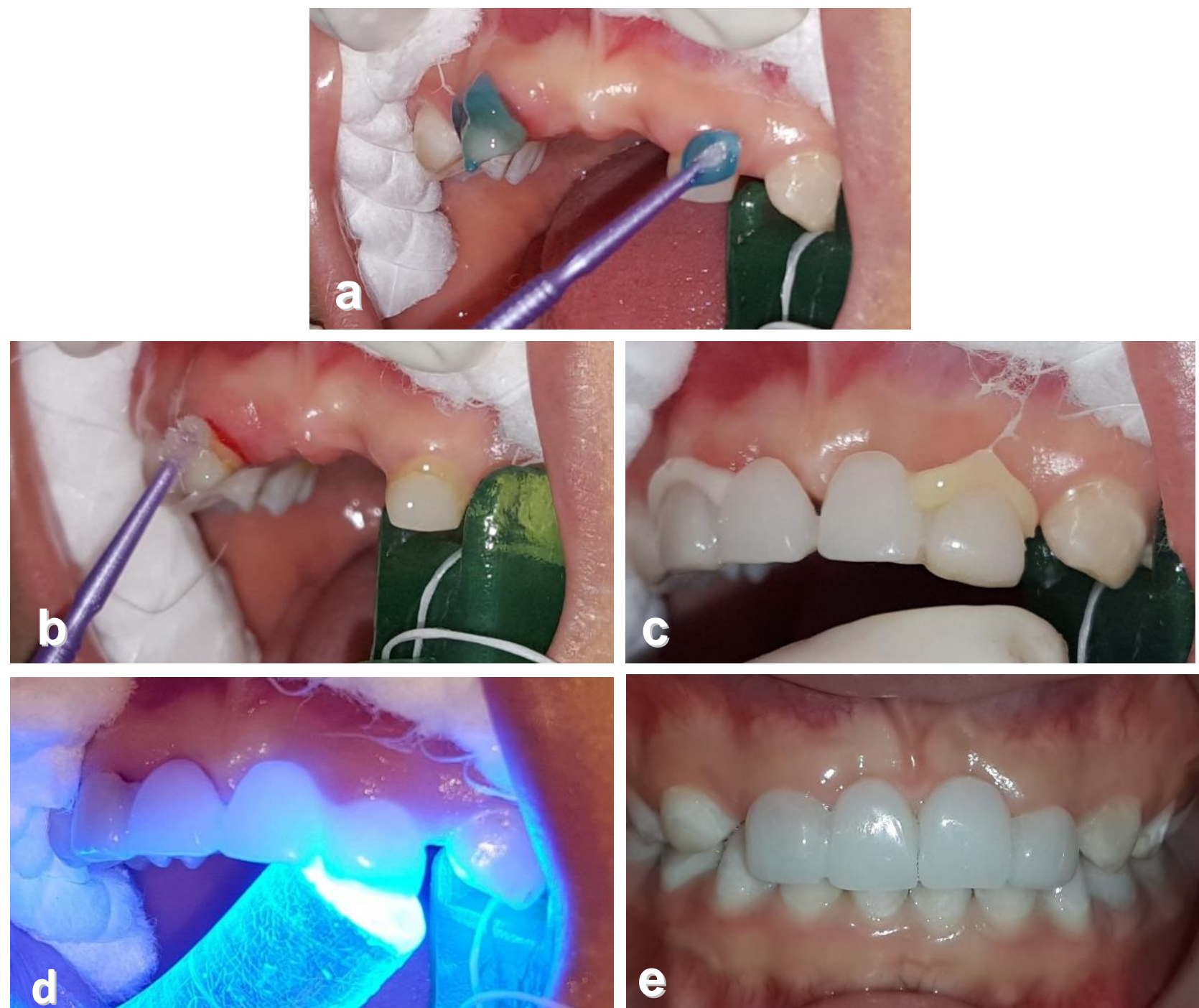

Figura 8. a) Acondicionamiento con ácido fosfórico. b) Colocación del adhesivo c) Inserción de la prótesis. d) Fotopolimerizado e) Vista frontal de la prótesis estético - funcional tipo Denari. 
Es Importante hacer mención, que la prótesis tiene fines estéticos, actúa de forma pasiva, por lo que no ejerce ninguna acción sobre los tejidos dentarios u óseos, cualquier modificación en la oclusión está dada por el crecimiento maxilo -facial normal del preescolar . En la parte postero -inferior se realizó la exodoncia de la pieza 7.4, por fracaso del tratamiento pulpar, es por este motivo que se colocó un mantenedor unilateral tipo banda - asa modificada, llevado al laboratorio y tratado de la misma manera que una prótesis metálica

colada, dado que la pieza 7.5 presenta una corona de Ivocron, motivo por el cual no se puede colocar una banda estándar de ortodoncia, ya que dificultaría su preparación, haciendo del mantenedor colado una buena para evitar la pérdida de espacio prematura, esto ayudara a evitar la mesialización de los dientes adyacentes a la perdida y la extrusión de los antagonistas. La (Figura 10) muestra el mantenedor fijo tipo banda - asa con buen ajuste sin daño en los tejidos adyacentes y su respectivo control de oclusión

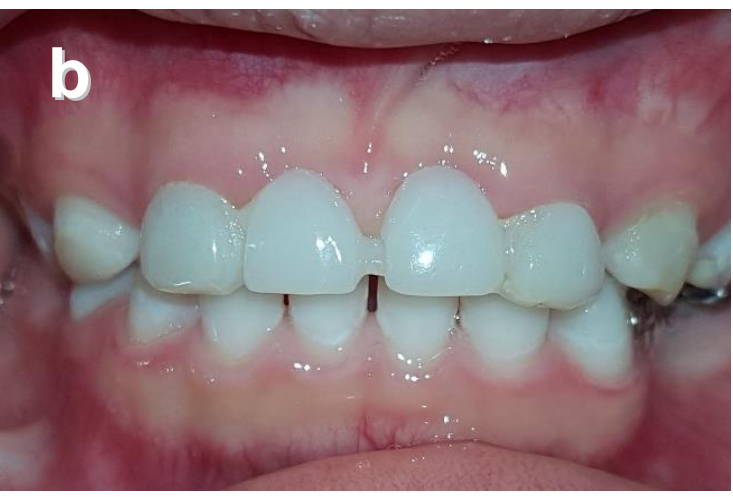

Figura 9. Vista antero -posterior de la prótesis fija estético - funcional tipo Denari, luego de 8 meses de seguimiento. a), b) Se aprecia el crecimiento transversal del sector anterior.
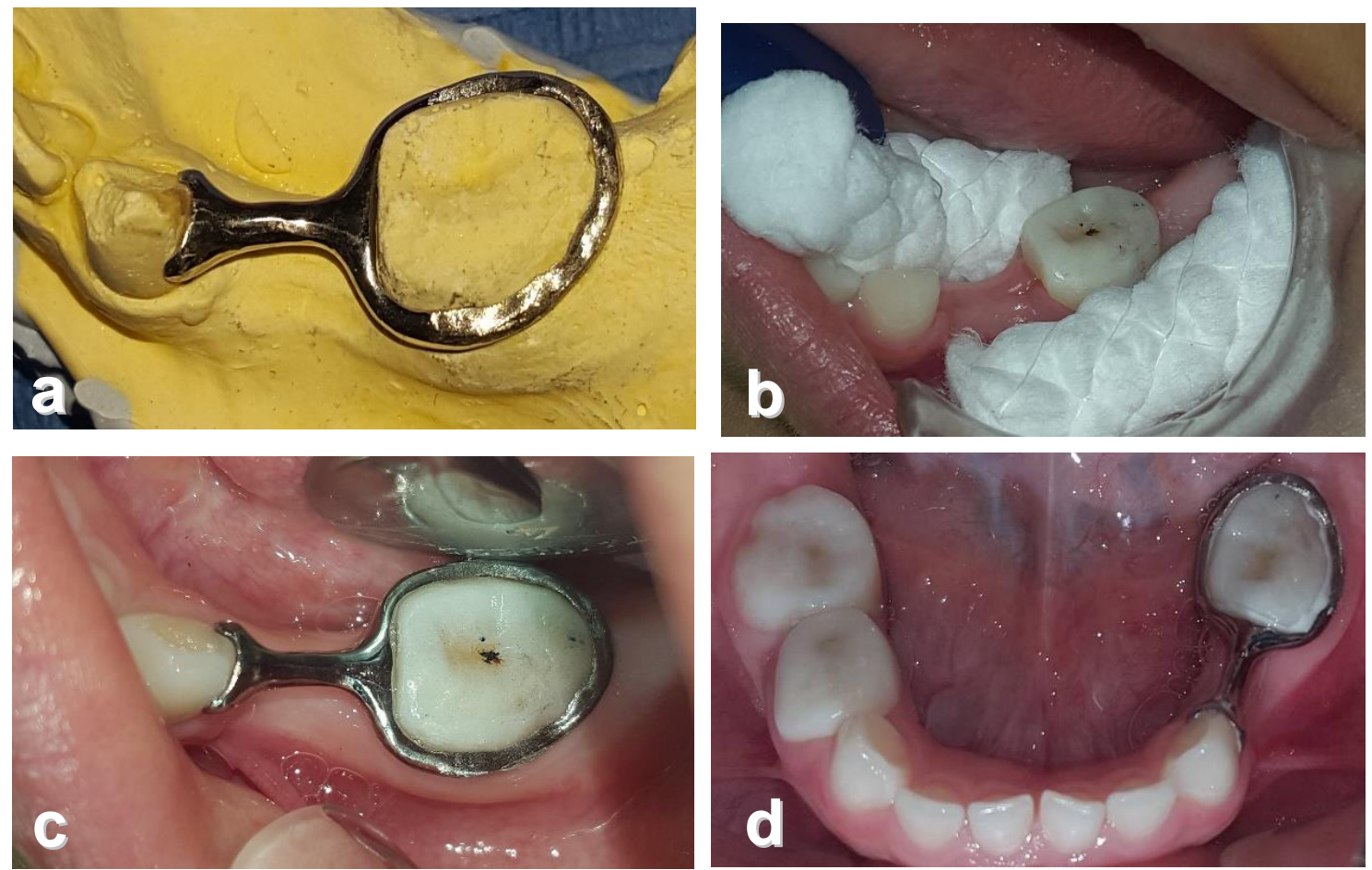

Figura 10. a) Confección del mantenedor colado en modelo de laboratorio. b) Aislamiento relativo c) Mantenedor cementado d) Vista oclusal. 
Durante el año de seguimiento la prótesis fue muy bien tolerada por el paciente, a pesar de su corta edad, el paciente asistió periódicamente a sus controles, en donde se evaluó la adaptación, salud de los tejidos blandos, mantenimiento de la prótesis y mantenedor de espacio respectivamente, solo pequeños ajustes fueron realizados. (Figura 11). Se envía al paciente a terapia de lenguaje en la espacialidad de motricidad oral para restablecer las funciones de deglución y fonación, un punto importante a destacar es el aumento de peso y talla del niño luego de restablecer la salud oral.
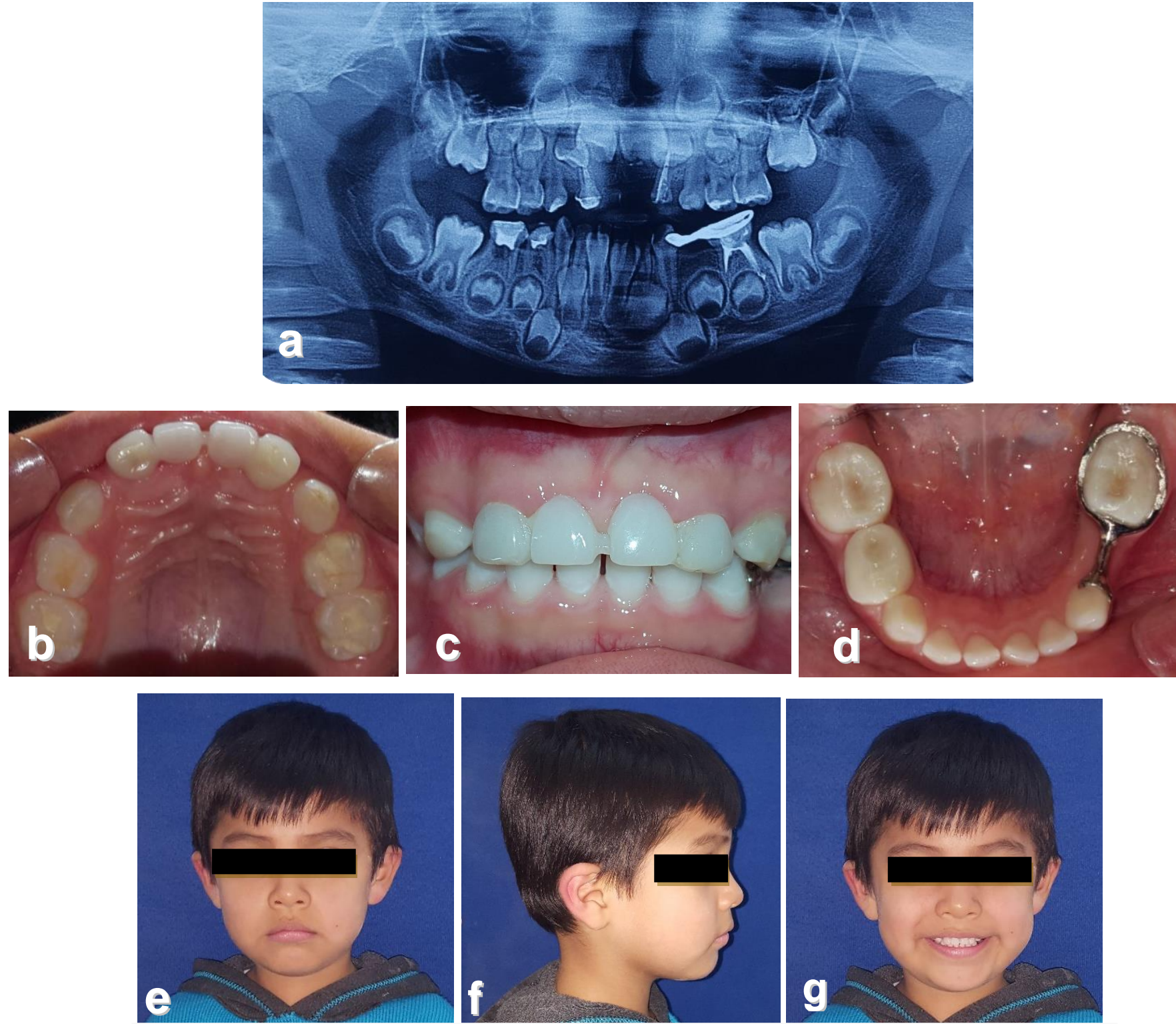

Figura 11. a) Imagen radiográfica después de un año de tratamiento.

Fotografías intraorales: b) vista palatina c) vista frontal d) vista lingual

Fotos faciales después de un año de tratamiento: e) frontal f) perfil g) sonrisa.

\section{DISCUSIÓN}

La pérdida prematura de los dientes anteriores deciduos es un problema frecuente en la clínica diaria de odontopediatría siendo los factores etiológicos más comunes el traumatismo dental, y la caries de infancia temprana $(\mathrm{CIT})$, relacionados al control de dieta cariogénica y de biofilm ${ }^{(10)}$. 
Mantener la integridad de las arcadas dentarias es necesario para una adecuada oclusión y correcto desarrollo de las funciones del sistema estomatognático.

Ante la pérdida prematura de cualquier diente primario, la principal preocupación es el desarrollo del sucesor permanente, por lo tanto el control del espacio rige los principios del tratamiento. Esto con la finalidad de evitar la mesialización de las piezas dentales hacia el espacio edéntulo, que a la larga puede producir maloclusiones como el apiñamiento, erupciones ectópicas, mordidas cruzadas, etc. ${ }^{(14)}$ Estas mesializaciones son más severas y se dan con mayor rapidez ante la pérdida de las molares deciduas, sin embargo en el sector anterior se mantiene estable ante una pérdida precoz, una vez que los caninos primarios han erupcionado ${ }^{11}$ Por lo tanto, al igual que en este caso clínico sigue siendo el deseo de los padres de devolver la estética la principal indicación descrita en la literatura para rehabilitar el sector anterior, seguida por las funciones masticatorias y pérdida de espacio ${ }^{(15,16)}$.

Para una correcta rehabilitación estético funcional del segmento anterior el profesional debe respetar las características morfológicas, funcionales y estéticas específicas de la dentición decidua, teniendo en consideración que una restauración en dientes deciduos es totalmente diferente a una restauración en dientes permanentes, la selección del color y del material tanto para procedimientos directos e indirectos, el tratamiento restaurador será bien aceptado si mantenemos una armonía en conjunto ${ }^{(17)}$.

Cada vez que se considera la necesidad de instalar un aparato protésico es importante considerar otros factores: a) edad del paciente para determinar el estadío y desenvolvimiento del sucesor permanente, cuanto menor fuese este estadío más indicado la sustitución de los dientes perdidos. b) número de dientes perdidos, c) capacidad de cooperación del paciente, d) etapa de desarrollo del lenguaje, función masticatoria y /o presencia de malos hábitos orales ${ }^{(18,19)}$.

En el presente caso clínico este último punto fue determinante para tomar la decisión de instalar un aparato protésico, a los pocos meses de haber perdido las piezas anteriores, el paciente presentó signos de interposición lingual. La ausencia de incisivos superiores hace que el niño explore con la lengua el espacio edéntulo, pudiendo provocar una protrusión maxilar o una lengua protráctil, la cual a su vez promueve el establecimiento de un patrón infantil de deglución con alteración de la actividad muscular y la consiguiente maloclusión (labioversión de incisivos y /o mordid abierta) ${ }^{(20,21)}$.

La pérdida de los incisivos a temprana edad puede interferir en el desarrollo de la fonoarticulación, aunque la literatura científica es controvertida al respecto, la producción de ciertos fonemas requiere que la lengua se apoye en las superficies palatinas de los incisivos superiores, cuando no están presente los incisivos ocurren compensaciones fonéticas durante la emisión de algunos fonemas. Por lo tanto es prudente considerar la instalación de algún aparato protésico en niños menores de 4 años que aún no han desarrollado el habla ${ }^{(19,22)}$.

La edad del paciente fue un factor importante para decidir optar por un dispositivo estético funcional fijo, ya que estos son menos molestos para el paciente y menos dañino para los tejidos orales que los dispositivos removibles, se evita las pérdida del aparato con consecuente gastos extras para los padres, y extrusión de los antagonistas. Varios autores concuerdan en que el mejor aspecto de una prótesis fija funcional es el hecho de que ofrece mayor seguridad, por lo tanto son las más apropiadas para largos periodos de uso. En el presente caso clínico se optó por una prótesis funcional fija modificada para su uso, porque el paciente se encuentra en el rango de edad indicado, no presentando la madurez y responsabilidad para usar una prótesis removible, que en ocasiones genera desconfort durante los primeros días, hecho que genera que el paciente deje de utilizar la prótesis en corto tiempo $(19,23,24)$

Hasta ahora no hay evidencia de calidad que reporte que los aparatos protésicos producen restricción del crecimiento en el niño según Graber, ${ }^{(20)}$ nos dice que después de la erupción de los temporales hay un periodo de descanso relativo y no hay cambio en los arcos hasta la erupción de los permanentes; sin embargo otros investigadores concluyen que en niños de 2 a 4 años de edad existe un incremento intercanino en el segmento superior en alrededor 0.2 a $1,5 \mathrm{~mm}$. $(13,22,25)$. el aumento es notorio en nuestro caso clínico, en menos de un año se obtuvo un crecimiento de $1,2 \mathrm{~mm}$, hecho que nos sugiere la necesidad de considerar alternativas protésicas con cierto dinamismo y que permitan acompañar el crecimiento maxilofacial del infante en el momento de rehabilitar el sector anterior. 
Es por ello que, el uso del aparato protésico semifijo como el dispositivo estético - funcional tipo Denari, en el presente caso permite prevenir la restricción del crecimiento transversal y evitar una probable mordida cruzada.

Hace cinco décadas, Finn ${ }^{(26)}$ sugirió que ante la ausencia temprana de los incisivos centrales deciduos, se podía construir un mantenedor no rígido y que permite la expansión fisiológica del arco en esta región. Para lo cual propuso adaptar bandas metálicas en los incisivos laterales, en los cuales por la cara mesial se suelda un tubo y un alambre grueso. Posteriormente la escuela brasilera Denari \& Correa en $1995^{(10)}$, preconiza el principio añadiendo un tubo-barra en la región palatina y sobre ello colocar piezas artificiales, tallando los dientes adyacentes para recibir las coronas que servirán de soporte para la prótesis, este dispositivo permite el desenvolvimiento normal de la maxila. 29.

Au ET. ${ }^{(27)}$ pone especial cuidado en la confección de la prótesis que el dispositivo no sea tan rígido para que haya crecimiento en lateralidad, la articulación permitirá que esto ocurra, basados en estos conceptos construimos un aparato protésico siguiendo el mismo principio, pero confeccionando el dispositivo de una manera más estética en Ivocron, la necesidad del tallado de los dientes adyacentes no fue un problema en este caso, ya que dichas piezas recibieron tratamiento pulpar previo y reconstruidos con un poste de fibra de vidrio, es por esta razón que la prótesis estético funcional fija tipo tubo - barra fue la elección en la rehabilitación en este caso.

La pérdida precoz de los incisivos deciduos puede traer influencias negativas desde el punto de vista psicológico ${ }^{(16)}$, los niños se sienten "diferentes" a sus compañeros de la misma edad, la falta de sus dientes empeora sus relaciones con otros niño y consigo mismos, especialmente se hace visible la falta de estos durante el habla y la sonrisa, la mayoría de veces el niño sonríe poco o se coloca la mano sobre la boca para disfrazar su falta, por eso es importante valorar el factor estético durante la perdida prematura, preservar el factor psicológico tanto del niño como el de los padres $^{(16,22)}$. La rehabilitación oral del presente caso tuvo una repercusión positiva en el incremento de la autoestima del paciente, mejorando sus relaciones interpersonales según lo referido por la madre.

Después de la colocación de la prótesis se dio orientación a los padres sobre la dieta, en la que se debe evitar alimentos excesivamente duros, en cuanto a la higiene es importante para preservar la integridad de la prótesis, así mismo se debe realizar el seguimiento radiográfico para determinar el momento de retirar la prótesis para la erupción de I

\section{CONCLUSIÓNES}

La prótesis fija estético - funcional modificada con sistema tubo - barra representa una alternativa rápida, cómoda para el paciente, mínimamente invasiva, estética ideal para rehabilitar la perdida precoz de los dientes anteriores deciduos, principalmente en la primera infancia. En el caso clínico descrito puede ser observado un resultado estético y funcional satisfactorio, que procura mejorar la salud bucal del paciente, restablecer psicológicamente a los padres y al niño. El paciente debe estar en constante acompañamiento para posibles necesidades de sustituir el aparato, reparar el desgaste, o la exfoliación de los dientes deciduos y evaluación de la higiene bucal. Teniendo en cuenta el impacto en la calidad de vida, el presente caso mostró resultados satisfactorios cumpliendo con restablecer la salud oral.

Contribuciones de autoría: SEM, MR y RVS participaron en el desarrollo del caso. Todos los autores aprobaron el artículo todos los autores

\section{Fuente de financiamiento:}

Autofinanciado.

\section{Conflicto de intereses:}

No existe conflicto de intereses en este manuscrito.

\section{REFERENCIAS}

1. Andreasen JO. Etiology and pathogenesis of traumatic dental injuries. Scand J dent Res 1970; 78(4):329-42.

2. Law CS. Management of premature primary tooth loss in the child patient. J Calif Dent Assoc. 2013;41(8):612-18.

3. Korytnicki Lanstein D, Naspitz N, Faltin Junior, Kurt. Consequências e tratamento das perdas precoces de dentes decíduos. Rev Assoc Paul Cir Dent. 1994;48(3):1323-28

4. Fierro C, Bravo L, Vera A, Torres M, Pérez A. Pérdida prematura de incisivos como factor agravante de hábitos, reporte de un caso clínico. Rev Soc Chil Odontopediatría. 2009;24: 35-8.

5. Baume LJ. Physiological tooth migration and its significance for the development of oclusion. The biogenetic course of the decíduos dentition. J Dent Res 1950;29(2):123-32.

6. Saini S, Sharma D. Functional and Esthetic Rehabilitation during Deciduous Dentition Stage: A Case Report. Dent Res J (Isfahan). 2011;8(2):10811 
7. Alsheneifi T, Hughes CV. Reasons for dental extrations in children further study. Pediatr Dent. 2001; 23(2):109-12.

8. American Academy Pediatric Dentistry. Guideline on management of the developing dentition and oclussion in pediatric dentistry. 2009. [consultado Oct 2016]. Disponible en: http://www.aapd.org/media/Policies Guidelines/G DevelopDentition.pdf

9. Rodrigues SA, Oliveira G, Marins D, GuedesPinto A. Primary anterior tooth replacement with a fixed prosthesis using a precision connection system. A case report. Quintessence Int. 2000;33: 303-8.

10. Diniz MB,Silva RC, Zuanon ACC, Perda dental precoce e manutencao de espacio na dentadura decidua:relato de un caso clínico .Rev ibero-am Odontopediatr Odontol Bebe 2005;8(44):376-8

11. Denari W, Correa D. Prótese parcial anterior pelo sistema tubo- barra. Rev Assoc Paul Cir Dent. 1995; 49:477-8.

12. Dominguez A. Aznar T, Removable prostheses for preschool children: report of two cases. Quintessence Int. 2004; 35(5): 397-400.

13. Gongalvest, L.M., et al. Uso de prótese fixa adesiva como mantenedor de espaço em dentes anteriores decíduos: um relato de caso. Archives of Oral Reseach, 2013; 9(1) 85-90.

14. De Sant'Anna GR, Guaré Rde O, Rodrigues CR, Guedes-Pinto AC. Primary anterior tooth replacement with a fixed prosthesis using a precision connection system: a case report. Quintessence Int; 2002; 33(4): 303-308.

15. Waggoner WF, Kupietzky A. Anterior esthetic fixed appliances for the preschooler: considerations and a technique for placement. Pediatr Dent. 2001; 23(2): 147-50.

16. Bijoor RR, Kohli K. Contemporary space maintenance for the pediatric patient. N Y State Dent J. 2005; 71(2): 32-35.
17. Pereira L, Miasato JM. Mantenedor de espaço estético-funcional em Odontopediatria: Rev Odontol Univ Cid São Paulo. 2010;22(2):154162.

18. Patil P., Rachoppa M. A simple modification of fixed space maintainers for replacement of an avulsed maxillary primary central incisor. International Journal of Dental Clinics, 2011; 3(1):117-20

19. Zaror Z, Hope B, Diaz J, Jans A. Proteisi fija con Sistema tubo-barra en odontopediatría: reporte de caso clínico de 12 meses de seguimiento. Rev Clin Periodoncia Implanto Rehabit Oral. 2015;8(3):239-243

20. Graber M.Ortodoncia Teoria a Practica 3 era Ed. Mexico Nueva Editorial Interamericana.1981.p 257-298.

21. Fathian M, Kennedy DB, Nouri MR. Laboratorymade space maintainers: a 7-year retrospective study from private pediatric dental practice. Pediatr Dent. 2007;29(6): 500-6.

22. Silva FWGP, Stuani AS, Queiroz AM. Importancia da manutencao de espaco em Odontopediatria. Odontologia.Cclin.-Ccientif., Recife 2007;6 (4): 289-292

23. Kapur A, Chawla HS, Goyal A, Gaube K. An esthetic point of view in very young children. J Clin Pediatr Dent. 2005; 30:99-103.

24. Paim S, Ribeiro A, Costa E, Modesto A. Prótese fixa adesiva: uma opção de tratamento para a perda precoce de incisivo decíduo. J Bras Odontopediatr Odontol Bebê. 1999;2(10):419-24.

25. Terlaje RD, Donly KJ. Treatment planning for space maintenance in the primary and mixed dentition. ASDC J Dent Child. 2001;68(2):109$14,80$.

26. Finn B. Sidney Odontología Pediátrica $4^{\text {ta }}$ Ed. México Nueva Editorial Interamericana 1982.

27. Au ET. The effect of premature loss of primary and permanent teeth. J Hawaii State Dent Asso9;2(2):9-12 